\title{
Autonomic neuropathy in patients with hepatic cirrhosis
}

\author{
B K Bajaj, M P Agarwal, B Krishna Ram
}

Postgrad Med J 2003;79:408-411

See end of article for authors' affiliations

\section{Correspondence to:}

Dr B K Bajaj, 26/33,

Ill-Floor, West Patel

Nagar, New Delhi

110008 , India;

docbajaj@yahoo.co.in

Submitted

6 November 2002

Accepted 7 March 2003

\begin{abstract}
Background: Autonomic neuropathy has been reported in patients with alcoholic liver disease but information on its occurrence in patients with non-alcoholic liver disease is contradictory.

Aim: To assess autonomic functions in patients with alcoholic and non-alcoholic liver disease.

Study design: Autonomic function using five standard tests was examined in 20 cirrhotics ( 10 alcoholics and 10 non-alcoholics) and 20 age and sex matched controls. The extent of autonomic dysfunction was determined in the patients and a comparison between the characteristics of patients with and without autonomic neuropathy was made.

Results: Sixteen $(80 \%)$ of the cirrhotic subjects were found to have evidence of autonomic neuropathy. Of these, three $(15 \%)$ patients had early parasympathetic damage, five $(25 \%)$ had definite parasympathetic damage, and eight $(40 \%)$ had combined (that is, both parasympathetic and sympathetic) damage. Nine $(90 \%)$ of the alcoholics and seven $(70 \%)$ of the non-alcoholics had autonomic dysfunction. Only one patient belonging to the alcoholic group had clinical evidence of peripheral neuropathy. Moreover, there was no significant association between subjective symptoms of autonomic neuropathy and objective evidence of autonomic damage as assessed by autonomic function tests. Autonomic dysfunction was significantly more frequent in advanced liver disease compared with early liver damage. Nine $(75 \%)$ out of 12 cirrhotic subjects belonging to Child class B and six $(85.7 \%)$ of the seven patients belonging to Child class $C$ had autonomic neuropathy.

Conclusion: This study shows that autonomic neuropathy is common in cirrhotic subjects, that it is found with comparable frequency in alcoholics and non-alcoholics, and that it increases in severity with increase in extent of liver damage, suggesting that liver damage contributes to the neurological
\end{abstract} deficit.

\section{PATIENTS AND METHODS}

The study was carried out on 20 patients with hepatic cirrhosis ( 10 alcoholics and 10 non-alcoholics) and 20 age and sex matched controls in the Department of Medicine, University College of Medical Sciences and GTB Hospital, Delhi, India. The diagnosis of cirrhosis was made histopathologically in all except two patients, in whom liver biopsy was contraindicated. In these two patients, the diagnosis was based on clinical examination, laboratory parameters, ultrasonographic findings, and the presence of oesophageal varices. The controls were healthy volunteers with no history of alcohol consumption and normal clinical and biochemical parameters. Subjects who were on drugs known to cause autonomic disturbance and those with a history suggestive of diabetes mellitus, ischaemic heart disease or other medical conditions, which could confound the interpretation of autonomic function tests in relation to cirrhosis, were excluded from the study. A detailed clinical history with special reference to symptoms of autonomic disturbance was taken from each subject and a thorough physical examination including neurological assessment was carried out. All the patients and the controls were subjected to a battery of five standard autonomic function tests as detailed below.

\section{Tests reflecting cardiac parasympathetic damage}

- Heart rate response to Valsalva manoeuvre

The subject was seated quietly and then asked to blow into a mouthpiece attached to a manometer, holding it at a pressure of $40 \mathrm{~mm} \mathrm{Hg}$ for 15 seconds while a continuous electrocardiogram (ECG) was recorded. The manoeuvre was repeated three times with one minute interval in between and results were expressed as:

Valsalva ratio $=$ longest $\mathrm{R}-\mathrm{R}$ interval after the manoeuvre $\div$ shortest R-R interval during the manoeuvre.

The mean of the three Valsalva ratios was taken as the final value.

\section{- Heart rate (R-R interval) variation during deep} breathing

The subject was asked to breathe deeply at six breaths/min (five seconds "in" and five seconds "out") for one minute. An ECG was recorded throughout the period of deep breathing and onset of each inspiration and expiration was marked on ECG paper. The maximum and minimum R-R intervals during each breathing cycle were measured with a ruler and converted to beats/min. The results of the test were expressed as the mean of the difference between maximum and minimum heart rates for the six measured cycles in beats/min. 
Table 1 Interpretation of autonomic function tests as normal, borderline, or abnormal depending on the value of the parameter measured

\begin{tabular}{|c|c|c|c|c|}
\hline Test & $\begin{array}{l}\text { Predominant } \\
\text { autonomic } \\
\text { function tested }\end{array}$ & Normal & Borderline & Abnormal \\
\hline Valsalva ratio & Parasympathetic & $>1.21$ & $1.11-1.20$ & $<1.10$ \\
\hline Deep breathing test (max-min heart rate beats/min) & Parasympathetic & $>15$ & $11-14$ & $<10$ \\
\hline Heart rate response to standing (30:15 ratio) & Parasympathetic & $>1.04$ & $1.01-1.03$ & $<1.00$ \\
\hline Blood pressure response to standing (fall in blood pressure in $\mathrm{mm} \mathrm{Hg}$ ) & Sympathetic & $<10$ & $11-29$ & $>30$ \\
\hline Blood pressure response to sustained hand grip (increase in diastolic pressure in $\mathrm{mm} \mathrm{Hg}$ ) & Sympathetic & $>16$ & $11-15$ & $<10$ \\
\hline
\end{tabular}

\section{- Immediate heart rate response to standing}

The test was performed with the subject lying quietly on a couch while the heart rate was recorded continuously on an electrocardiograph. The patient was then asked to stand unaided and the point at starting to stand was marked on ECG paper. The shortest R-R interval at or around the 15th beat and the longest R-R interval at around the 30th beat after starting to stand were measured with a ruler. The characteristic heart rate response was expressed by 30:15 ratios

\section{Tests reflecting sympathetic damage}

\section{- Blood pressure response to standing}

This test measured the subject's blood pressure with a sphygmomanometer while he was lying quietly and one minute after he was made to stand up. The postural fall in blood pressure was taken as the difference between the systolic pressure lying and the systolic blood pressure standing. The test was repeated three times and the mean was calculated.

\section{- Blood pressure response to sustained hand grip}

The blood pressure of the patient was taken three times before the manoeuvre. A modified sphygmomanometer was used for sustained handgrip manoeuvre. The patient was asked to grip the inflatable rubber bag and apply maximum voluntary pressure possible. A reading from the attached mercury manometer was taken during maximum voluntary contraction. Thereafter, the patient was asked to maintain $30 \%$ of maximum voluntary contraction for as long as possible up to five minutes. Blood pressure was measured at one minute intervals during the handgrip. The result was expressed as the difference between the highest diastolic blood pressure during the handgrip exercise and the mean of the three diastolic blood pressure readings before the handgrip began.

Interpretation of tests was based on the works of Ewing and Clarke (table 1). ${ }^{6}$ The patients were categorised as normal, if none of the tests was abnormal; with early parasympathetic damage, if results of one of the three tests of parasympathetic function was abnormal; with definite parasympathetic damage, if two or more of the three tests of parasympathetic function were abnormal; and with combined damage, if one or both the tests of the sympathetic function were abnormal in addition to parasympathetic damage. For the purpose of the above mentioned classification the borderline tests were interpreted as normal.

A scoring system like the one suggested by Bellavere et al was also utilised to assess the extent of autonomic nervous damage. . For each test " 0 " score was given for normal, " 1 " for borderline, and "2" for an abnormal value. By adding the score of each of the five standard tests of autonomic function, total autonomic function score was determined for every subject. A comparison of frequency of symptoms of autonomic neuropathy was made between cirrhotics and controls, and between alcoholic and non-alcoholic groups. The relation of the presence of symptoms of autonomic neuropathy with abnormal autonomic function tests was determined using the $\chi^{2}$ test. Comparison of characteristics of patient with and without autonomic neuropathy was done using the same test. A simple set of clinical and laboratory features as devised by Child and Turcotte (later modified by Pugh and named ChildPugh criteria) were used in the study to quantify the severity of liver damage in patients. Scoring is done on the basis of degree of ascites, encephalopathy, hypoalbuminaemia, hyperbilirubinaemia, and hypoprothrombinaemia. The score of each of the parameters in an individual is added to classify a patient as belonging to Child class A, B, or C (table 2). This grading of cirrhosis was originally devised to help select patients with cirrhosis for portal systemic shunt surgery and it has been shown to have prognostic value in several studies.

\section{RESULTS}

The present study included 20 age and sex matched patients with hepatic cirrhosis (10 with a history of alcohol intake more than $80 \mathrm{~g} /$ day for at least eight years, and the remaining 10 with no history of ingestion of alcohol) and 20 controls $(p<0.05)$. None of our patients was found to have sympathetic dysfunction alone. Parasympathetic damage was found to be always present whenever there was evidence of sympathetic damage (table 3). The mean of the total autonomic function score was found to be significantly different in patients and controls $(\mathrm{p}<0.01)$. The mean $(\mathrm{SD})$ total autonomic function scores in cirrhotic and controls were 5.30 (1.84) and 0.30 (0.57) respectively. Total autonomic function scores in alcoholics, 5.90 (1.73), and non-alcoholics, 4.70 (1.83), were not found to be significantly different $(\mathrm{p}=1.49)$. 
Table 3 Distribution of autonomic dysfunction among cirrhotic groups

\begin{tabular}{lllll}
\hline & \multicolumn{2}{l}{ Parasympathetic damage } & Sympathetic \\
Patient group & Early & Definite & $\begin{array}{l}\text { Combined } \\
\text { damage }\end{array}$ \\
\hline Total cirrhotics $(n=20)$ & 3 & 5 & 8 & 8 \\
Alcoholics $(n=10)$ & 1 & 3 & 5 & 5 \\
Non-alcoholics $(n=10)$ & 2 & 2 & 3 & 3 \\
\hline
\end{tabular}

Table 4 Distribution of autonomic dysfunction in patients according to their Child class

\begin{tabular}{llll}
\hline Group & $\begin{array}{l}\text { Child class } \\
\mathrm{A}(\mathrm{n}=1)\end{array}$ & $\begin{array}{l}\text { Child class } \\
\mathrm{B}(\mathrm{n}=12)\end{array}$ & $\begin{array}{l}\text { Child class } \\
\mathrm{C}(\mathrm{n}=7)\end{array}$ \\
\hline $\begin{array}{l}\text { Early parasympathetic } \\
\text { damage }\end{array}$ & 1 & 2 & 0 \\
$\begin{array}{l}\text { Definite parasympathetic } \\
\text { damage }\end{array}$ & 0 & 3 & 2 \\
$\begin{array}{l}\text { Combined damage } \\
\text { Total }\end{array}$ & 0 & 4 & 4 \\
\hline
\end{tabular}

In the present study, nine (75\%) out of 12 patients belonging to Child class B had autonomic dysfunction while six $(85.7 \%)$ of the seven patients belonging to class $\mathrm{C}$ had impaired autonomic function. Only one patient with Child class A had early parasympathetic damage (table 4). The mean total autonomic function score was significantly lower $(\mathrm{p}<0.05)$ in Child class B $(4.58)$ patients than in those belonging to class $\mathrm{C}(6.71)$.

Heart rate response to standing was found to be the most frequently ( 11 out of 20 patients) abnormal test in cirrhotics. Eight patients had abnormal heart rate response to deep breathing, eight had abnormal blood pressure response to sustained handgrip, and seven patients had an abnormal Valsalva ratio.

Only one patient had clinical evidence of peripheral neuropathy in the study. No significant association between symptoms of autonomic dysfunction and objective evidence of autonomic neuropathy, as assessed by the five tests of autonomic function used, was observed. Five of the eight patients who did not report light headedness on standing had abnormal autonomic function test(s). Moreover, blood pressure response to standing was not abnormal in any patient including those with complaint of light headedness on standing. However, the results of the blood pressure response to standing were in the borderline range for 11 of the cirrhotics.

\section{DISCUSSION}

Autonomic nervous dysfunction is a known complication of diabetes $^{68}$ and alcohol abuse. ${ }^{10}$ Autonomic damage is expected in some patients with alcohol related cirrhosis since autonomic neuropathy, especially of vagal origin, is seen in chronic alcoholics. ${ }^{11}$ Evidence for vagal neuropathy in alcoholic cirrhosis is well established by various studies. ${ }^{2}{ }^{12}$ However, in non-alcoholic cirrhosis there are conflicting reports. ${ }^{12}{ }^{13}$ Only a few published Indian studies are available on the subject. ${ }^{14}$

In our study, 16 of the 20 cirrhotics $(80 \%)$ were found to have an abnormal result in one or more autonomic function tests. However, Barter and Tanner in their study of 30 subjects reported evidence of parasympathetic damage in 16\% and of combined parasympathetic and sympathetic neuropathy in an additional $20 \%{ }^{2}$ The lower frequency of autonomic dysfunction in their study could be due to the fact that they included only 14 subjects with alcoholic liver disease while the rest had an alcohol dependence problem only. Szalay et al in their evaluation of 121 patients with chronic alcoholism-33 without liver disease, 33 with fatty liver, 33 with cirrhosis, 10 with biliary cirrhosis, and 12 with cirrhosis of another originfound autonomic reflex damage in all. ${ }^{3}$ They observed significantly more damage in those with liver disease. Hendrickse and Triger reported cardiovascular autonomic dysfunction with predominantly parasympathetic abnormality in $35 \%$ of the patients with chronic liver disease. ${ }^{15}$

Hendrickse et al in another study reported vagal neuropathy in $45 \%$ of the 60 patients of chronic liver disease studied.$^{16}$ The lower frequency of neuropathy is probably due to inclusion of mostly Child class A patients in the study (57 of the 60 patients). Moreover, the study included a heterogeneous group of chronic liver disease patients with varying degrees of liver damage. In the present study, only one patient belonged to Child class A and the rest were class B or C. Gentile et al found autonomic neuropathy in $60 \%(71 \%$ in the alcoholic group and 57\% in the non-alcoholic group) of the 113 cirrhotics studied. ${ }^{17}$ Like in the present study, alteration of parasympathetic function was significantly more frequent than that of sympathetic function. Dillon et al also detected abnormal cardiovascular reflexes in $60 \%$ of 70 cirrhotics. $^{18}$ Their study group included as many as 42 patients belonging to Child class A and only 15 patients in class $C$.

Gonzalez-Reimers et al have remarked in a study that in alcoholics, autonomic and peripheral neuropathy are dependent on each other and there is only weak correlation between liver function and both autonomic and peripheral neuropathy. ${ }^{5}$ In the present study no apparent relationship was noticed between autonomic and peripheral neuropathy, and autonomic damage was observed to be more common than peripheral neuropathy. The main object of the present study was to examine autonomic neuropathy and so, while careful systematic clinical examination was done in all the subjects, it is probable that more sensitive electrophysiological measurement would have yielded a much higher proportion of peripheral nerve abnormalities.

In the present study, nine (75\%) out of 12 patients belonging to Child class B had autonomic dysfunction while six $(85.7 \%)$ of the seven patients in class C had impaired autonomic function. Only one patient in Child class A had early parasympathetic damage. The mean total autonomic function score was 4.58 in Child class B and 6.71 in class C. These findings are similar to the observations of most other studies, which reported increasing frequency of autonomic dysfunction with increasing severity of liver damage. Hendrickse and Triger reported a strong correlation between the abnormal tests and Child-Pugh score $(\mathrm{p}<0.0001) .{ }^{15}$ In their study, they found autonomic dysfunction in $69 \%$ of Child class B and C patients and $23 \%$ in class A patients $(p<0.0001)$. On the contrary, Gonzalez-Reimer et al in their study of 33 alcoholics, 20 of them cirrhotics, found a weak correlation between liver function and both autonomic and peripheral neuropathy.

Statistical comparison of cirrhotics and controls and alcoholics and non-alcoholics revealed that light headedness on standing was significantly more frequent in cirrhotics compared with controls $(p=0.001)$. However, no statistically 
significant association was noted between other symptoms or signs of autonomic dysfunction. This is similar to the findings of most of the studies available which found poor correlation between symptoms of autonomic neuropathy and objective evidence of autonomic neuropathy as assessed by the autonomic function tests. In our study, no statistically significant difference was observed for various clinical features and laboratory parameters of liver failure between those with and without autonomic neuropathy. This is in contrast to the findings of Hendrickse et al who observed that patients with vagal neuropathy were significantly older and tended to have lower serum albumin than those with normal cardiovascular test. ${ }^{16}$

In our study, heart rate response to standing was the most frequently ( 11 out of 20 patients) abnormal test in cirrhotics. Barter and Tanner in their study noted the heart rate response to standing as the most sensitive test with high specificity. ${ }^{2}$ Thuluvath and Triger in their study reported the heart rate response to deep breathing as the most sensitive test. ${ }^{4}$ However, it is noteworthy that this test depends on the cooperation of the subject and is, thus not as reproducible as the heart rate response to standing. Gentile et al remarked that deep breathing test and handgrip tests are the most influenced by the compliance of the patient. ${ }^{17}$ In the study, they found the deep breathing test and lying to standing (heart rate response) tests to be most altered and the most sensitive and specific tests respectively. In the present study, eight patients had abnormal heart rate response to deep breathing, eight had abnormal blood pressure response to sustained handgrip, and seven patients had an abnormal Valsalva ratio.

Finally, considering the adverse prognostic implications of autonomic neuropathy reported in cirrhotics, further prospective studies involving a larger number of patients are warranted to delineate the factors responsible for the derangement and find remedial measures if possible.
Authors' affiliations

B K Bajai, M P Agarwal, B Krishna Ram, Department of Medicine, UCMS-GTB-Hospital, Shahdara, Delhi, India

\section{REFERENCES}

1 Hendrickse MT, Triger DR. Autonomic dysfunction in chronic liver disease. Clin Auton Res 1993;3:227-31.

2 Barter $\mathbf{F}$, Tanner AR. Autonomic neuropathy in alcoholic population. Postgrad Med J 1987;63:1033-6.

3 Szalay F, Oravecz L, Kadar E, et al. Autonomic neuropathy in chronic liver disease. Gastroenterology 1990;50:187-9.

4 Thuluvath PJ, Triger DR. Autonomic neuropathy and chronic liver disease. QJ Med 1989:72:737-47.

5 Gonzalez-Reimers E, Alonso-Socas M, Santolaria-Fernandez F, et al. Autonomic and peripheral neuropathy in chronic alcoholic liver disease. Drug Alcohol Depend 1991;27:219-22.

6 Ewing DJ, Clarke BF. Diagnosis and management of diabetic autonomic neuropathy. BM 1982;285:916-19.

7 Bellavere F, Bosello G, Fedele D, et al. Diagosis and management of diabetic autonomic neuropathy [letter]. BM 1983;287:61.

8 Ewing DJ, Campbell IW, Clarke BF. Assessment of cardiovascular effects in diabetic autonomic neuropathy and prognostic implications. Ann Intern Med 1980;92:308-11.

9 Novak DJ, Victor $M$. The vagus and sympathetic nerves in alcoholic polyneuropathy. Arch Neurol 1974;30:273-84

10 Johnson RH, Robinson BJ. Mortality in alcoholics with autonomic neuropathy. J Neurol Neurosurg Psychiatry 1988;51:476-80.

11 Duncan G, Johnson RH, Lambie DJ, et al. Evidence of vagal neuropathy in chronic alcoholics. Lancet 1980;ii: 1053-7.

12 Decaux G, Cauchie P, Soupart A, et al. Role of vagal neuropathy in the hyponatraemia of alcoholic cirrhosis. BM 1986;293:1534-6.

13 Lenz K, Hortnagl, H, Magometschnigg, D, et al. Functions of the autonomic nervous system in patients with hepatic encephalopathy. Hepatology 1985;5:831

14 Khosla SN, Sanyal S, Nand N. Autonomic function tests and clinical significance of dysautonomia in chronic liver disease. J Assoc Physicians India 1991;39:924-6.

15 Hendrickse MT, Triger DR. Autonomic dysfunction and hepatic function in chronic liver disease. Gut 1990;31:A1 164

16 Hendrickse MT, Thuluvath PJ, Triger DR. Natural history of autonomic neuropathy in chronic liver disease. Lancet 1992;339:1462-4.

17 Gentile S, Marmo R, Peduto A, et al. Autonomic neuropathy in liver cirrhosis: relationship with alcoholic aetiology and severity of disease. Ital J Gastroenterol 1994:26:53-8.

18 Dillon JF, Plevris JN, Nolan J, et al. Autonomic function in cirrhosis assessed by cardiovascular reflex tests and 24 -hour heart rate variability. Am J Gastroenterol 1994;89:1544-7. 